\title{
A SIMULATOR FOR HELPING IN DESIGN OF A NEW ACTIVE CATHETER DEDICATED TO COLOSCOPY
}

\author{
Dumont, G. \& Kuehl, C. \\ IRISA Rennes, equipe SIAMES, Campus de Beaulieu, F-35042 Rennes Cedex, France \\ E-mail: georges.dumont@irisa.fr
}

\begin{abstract}
In the paper a dynamical simulator of an active micro-catheter for coloscopy is presented. A brief description of a real prototype is proposed and outlined the interest of designing a training simulator. The techniques of virtual prototyping aimed at improving the quality of the developed prototypes. The quality of the model was evaluated by an objective function. In order to minimise the objective function, which leads to a better device, different optimization approaches by genetic algorithms have been used. A simulator based on a mechanical description of the device and on the interacting environment allows computing the progression of a poly-articulated endoscope.

(Accepted by previous Editorial Team.)
\end{abstract}

Key Words: Dynamical Simulator, Catheter, Genetic Algorithms, Optimisation

\section{INTRODUCTION}

In this paper we focus on a dynamical simulator dedicated to the global design, by means of virtual prototyping methods, of an active micro-catheter for coloscopy. These devices are fully in the scope of surgeons demand for near future, because they enable the operating gesture to be assisted [1] and thus, minimise one patient hurt during intervention. Prototypes of such systems are proposed for more than ten years [2], but only few works are dedicated to the computer aided design process of these devices [3, 4]. Classic flexible endoscopes are generally constituted of articulated links, actuated by four cables connected to the head of the endoscope. The motion is achieved by means of knurls that are pulled by the surgeon, so the gesture is not assisted. A new generation of active endoscopes was developed by Olympus [5]. In this device, the head orientation is controlled through flexion modules activated by SMA actuators. However, this system does not automatically adapt its curvature to the environments. Developments of new prototypes, aiming at improving the navigation of endoscopes in the inspected ducts, are in progress. Prototypes constituted of trays interconnected by SMA threads have been proposed in which the piloting the length of the threads controls the orientation between two consecutive trays. One, for example, has been proposed in [6]. Its diameter is $8 \mathrm{~mm}$ and only the head is controlled. Another has been proposed in [7]. This prototype is of diameter $1.7 \mathrm{~mm}$ and is dedicated to interventions in ureter. It is presented in Fig. 1.

We propose here a brief description of our real prototype and outline the interest of designing a training simulator. As the foreseen catheter will have to crawl its way inside complex environments as human ducts, we have chosen a modular stiff poly-articulated structure presented in Fig. 2 and designed in our laboratories [8, 9].

The chosen mechanism consists in a succession of modules related to each other by pin joints, which are alternatively oriented at $90^{\circ}$. This configuration allows a 3D motion of the structure. The endoscope head should contain a device allowing obtaining a multi-directional vision of the observed space. This will be achieved by using a polymer gel actuated prism. 


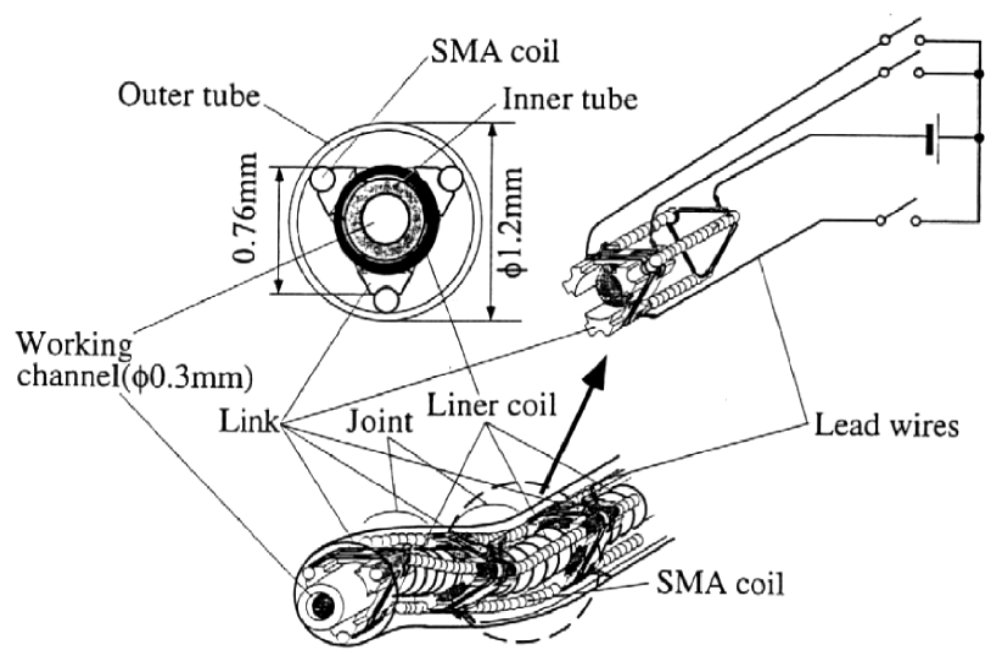

Figure 1: Multilink active catheter [7].

As medical device, a flexible polymer sheath should protect the whole system. Each joint is provided with two antagonist electrically commanded SMA spring-like actuators allowing the control of the relative orientation of consecutive links. An integrated circuit controlling the electrical power supplied to the SMA realizes the command. The SMA actuators have been chosen because they ensure a good compromise between their mass and the forces that they can deliver.

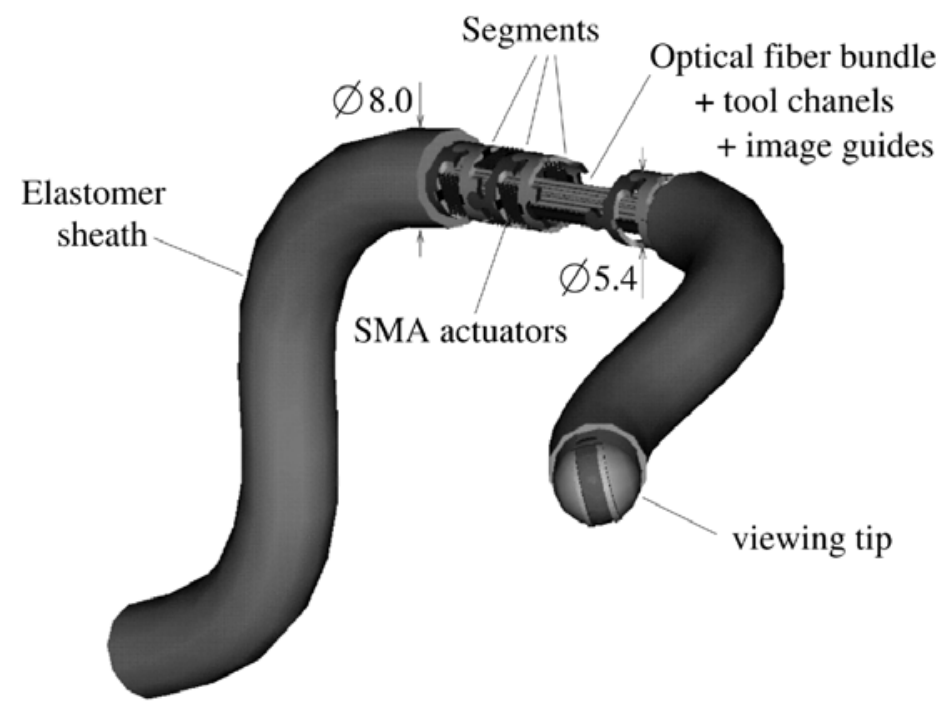

Figure 2: Schematic view of the prototype.

Fig. 3 presents our simulator. The objective for this simulator is to realize virtual operations in a virtual patient body, modelled by a reconstruction of the digestive tract. In our case, the model of this digestive track is obtained by Magnetic Resonance Imaging (MRI). The surgeon feelings will be maximal by using graphic and haptic interfaces provided by the virtual reality techniques. The computational model used for the simulation is the model of the proposed real prototype.

The interests for a design and training simulator are triple:

- Teaching to young surgeons: the training on simulator is obviously more accessible, less expensive and less risky and should allow to experiment pathologic cases; 
- Preoperative training: the simulator will allow the surgeon to virtually repeat the operation on the patient, before the real operation and thus minimize the risks;

- Virtual prototyping [10]: furthermore, the simulator allows to virtually test the tool quality with respect to a given operation task. By using optimization techniques based on genetic algorithms, we can test which is the best candidate to achieve this task, and propose an adapted endoscope.

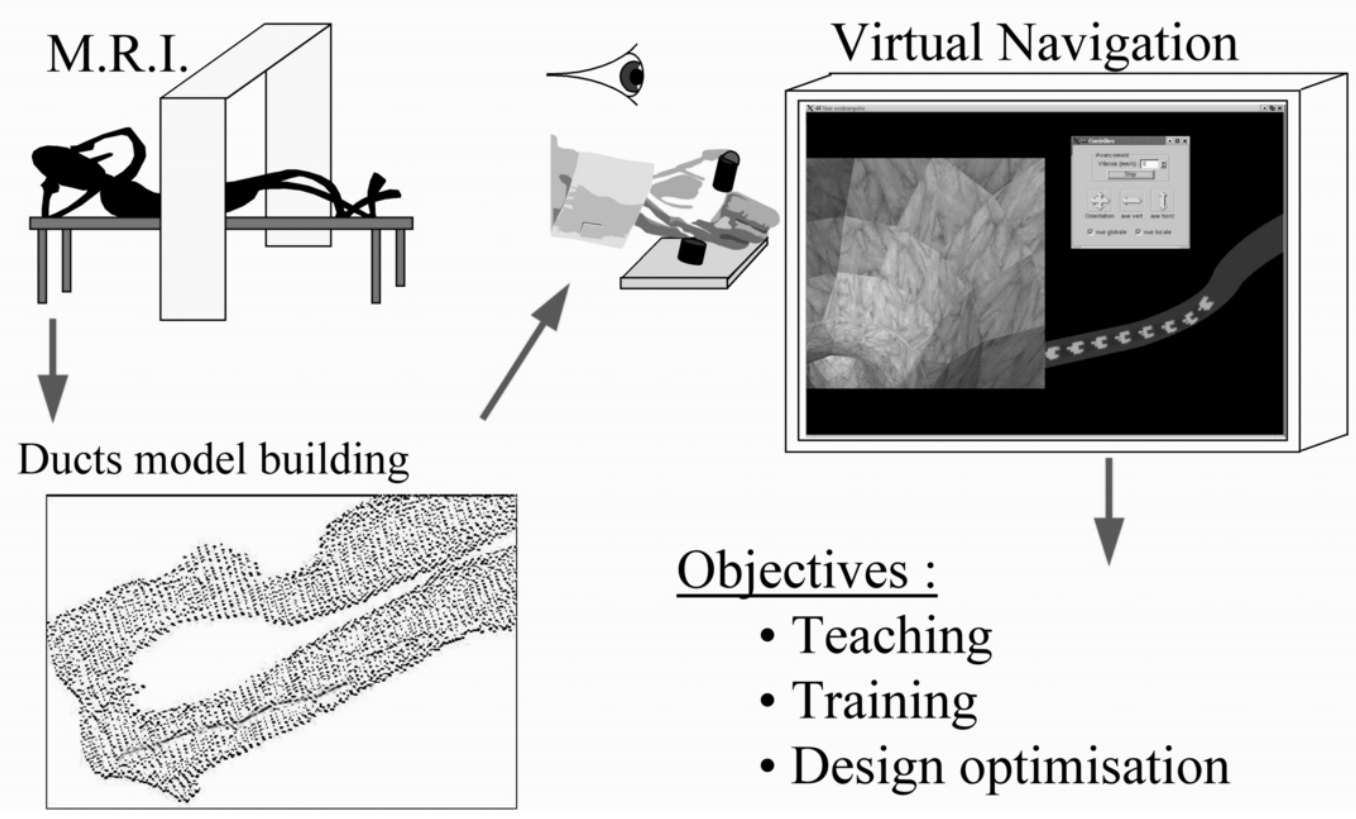

Figure 3: Proposed Simulator.

In the following, we will focus on the simulator components. In order to deal with endoscope toward ducts interaction, we present the "devoxelisation" process, which leads to an interpolated distance cartography of the ducts. This database construction is used to minimize the computation time for treatment of the interactions. The contact detection algorithm, allowing determining these interactions forces between the endoscope model and the duct model is then presented. Let us notice that an important experimental work should be lead to identify the parameters of this contact model. A result will illustrate the simulation process. To minimize one patient hurt during operation, we use the control capability of the device. We will address it by a multi-agent control strategy for controlling the catheter conformation to the ducts and compare this method to the uncontrolled case. It is shown that the magnitude of contact forces decreases.

The third proposed use of the simulator is the design optimization of the prototype. To address this purpose, the simulator is coupled to genetic based algorithms. This allows showing the feasibility of an optimization process for geometric conditions, where the design is optimized with respect to accessibility conditions. We propose also an optimization process for dynamic conditions, where a dynamical simulation of the system evaluates the design during the realization of an inspection task in the environment.

\section{DESCRIPTION OF THE SIMULATOR}

A mechanical model of the proposed real prototype allows us to better understand its capabilities. The model is a kinematical open chain described by a Denavit and Hartenberg representation presented in Fig. 4. 


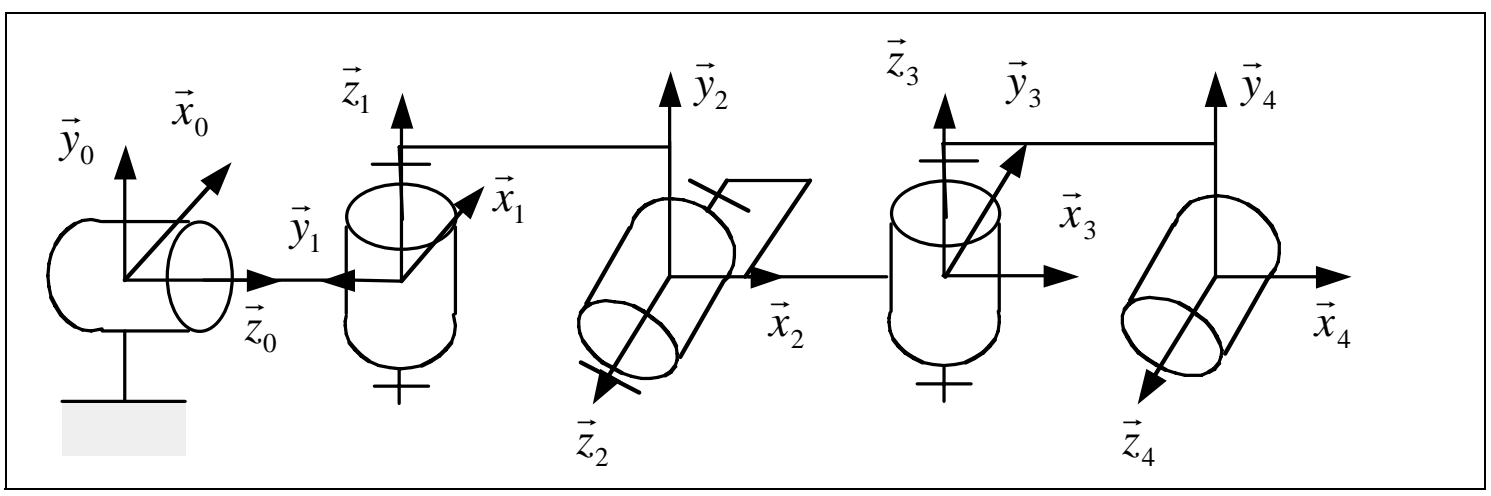

Figure 4: Denavit-Hartenberg representation of the endoscope.

Let $\left(q_{i}\right)=\left(z_{1}, \theta_{1}, \theta_{2}, \ldots, \theta_{n}\right)$ denote the set of parameters describing the endoscope configuration. The Lagrange formulae allows to write the motion equation for such a model:

$$
\frac{d}{d t} \frac{\partial K_{E}}{\partial \dot{q}_{i}}(q, \dot{q}, t)-\frac{\partial K_{E}}{\partial q_{i}}(q, \dot{q}, t)=Q_{i}-\frac{\partial E_{p}}{\partial q_{i}}-\frac{\partial E_{d}}{\partial \dot{q}_{i}}
$$

In this equation, $K_{E}$ is the kinetic energy of the system. As in our application, the movements are slow and as the mass of the device is small, we neglect this inertia effect in the model.

In this equation, $Q_{i}$ are the external forces due to contact interactions and $E_{p}$ denotes the potential energy due to gravity $E_{\text {grav }}$ and elasticity $E_{p e}=\frac{1}{2} K_{s}\left(\theta_{i}-\theta_{i 0}\right)^{2}$ of the polymer sheath. The dissipative term at each pin joint is written as $\frac{\partial E_{d}}{\partial \dot{\theta}_{i}}=A \dot{\theta}_{i}$.

The obtained equations [11] are written as:

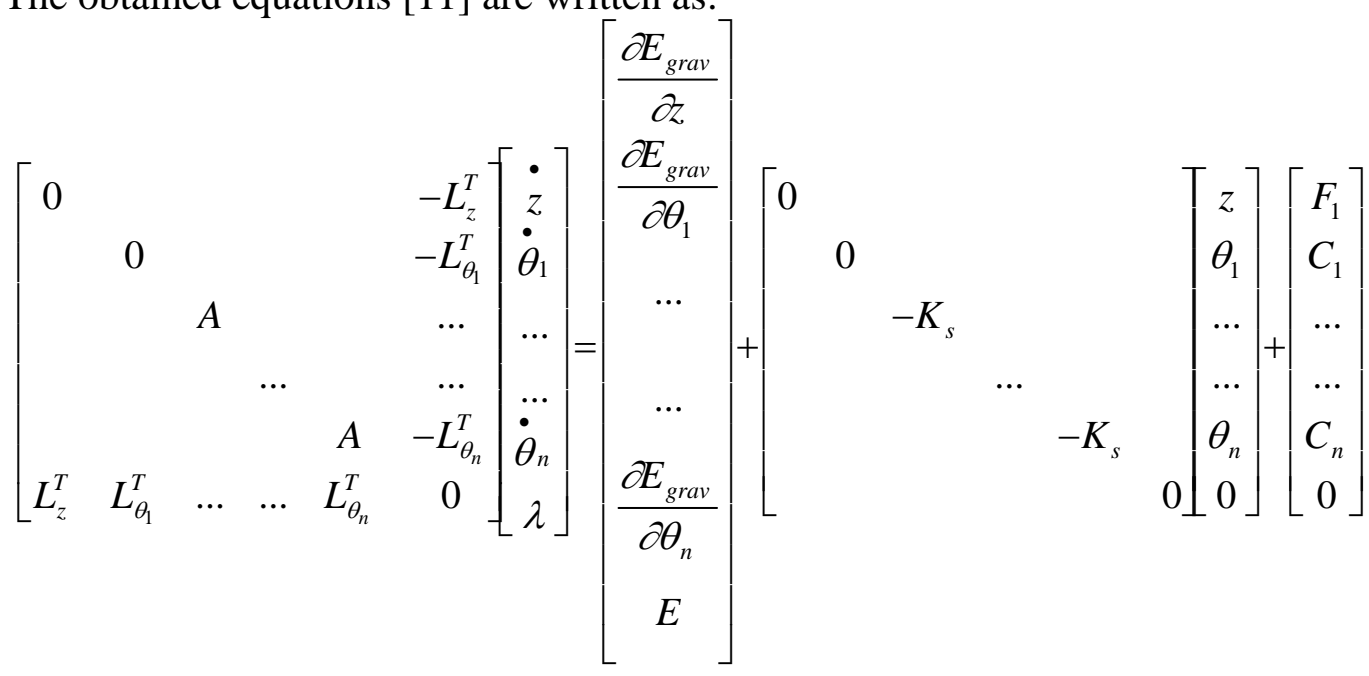

They may be rewritten in a more compact equation as:

$$
A \bullet \dot{q}=G(q, t)+K_{s} \bullet\left(q-q_{0}\right)+F(q, \dot{q}, t)
$$


In this expression, $G$ represents the gravity effect, $K_{s}$ represents the elasticity effect of the SMA actuators in uncontrolled configuration and $F$ represents the forces exerted by the SMA actuators in the controlled configuration and the contact forces. The terms related to the gravity effect and to the external forces are not constant; we need to evaluate them at each time step.

These equations constitute a first order differential equation system. The choice of the integration method for solving this system is important to ensure the convergence of the algorithm. As our models are constituted of long open chains, we have to use a precise and stable method. Thus, as the number of links may increase, the time step will have to be reduced. Furthermore, the use of this computational model into an optimization process, will lead the algorithm to compute model of variable length. For all these reasons, we are using a Runge-Kutta algorithm (of $4^{\text {th }}$ order) with adaptive time-step as proposed in [12].

The management of the simulator is implemented on the OpenMASK† platform [13] developed at IRISA. The main objective of OpenMASK is to propose a modular simulation to be executed on various material configurations. The platform manages the synchronization and the exchanges of data between the co-operative processes insuring a "dilated real time" in its standard version. It is developed by using the specificities of the object-oriented programming. The synoptic of its organization for our application is presented in Fig. 5.

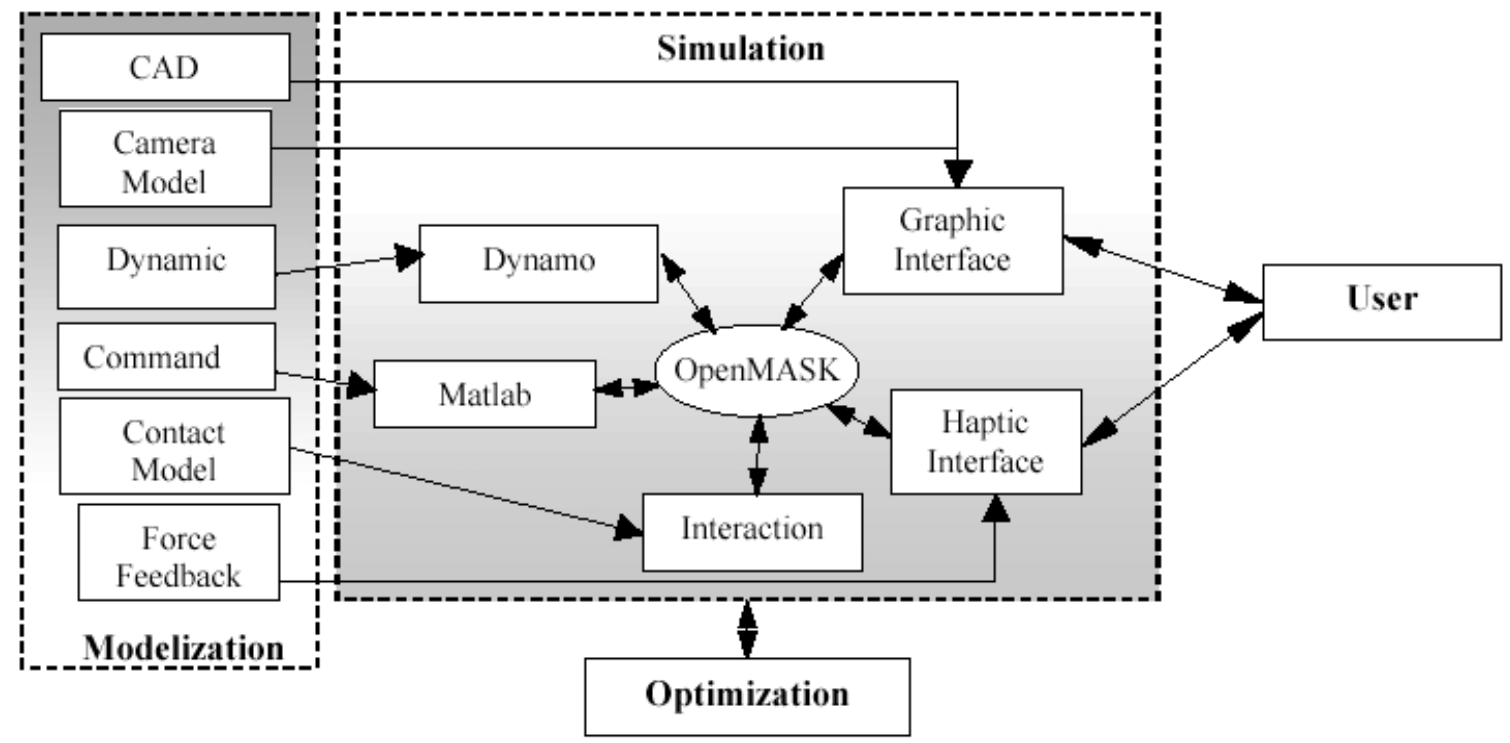

Figure 5: OpenMASK: Modular Animation and Simulation Kit.

Various models [14] are proposed to describe the behaviour of human organs or tissues, including non-linear behaviour and relaxation. A first approach, because the interaction occurs between a stiff body imposing the motion (the links of the endoscope on one hand) and a soft body (human tissues on the other hand), is to compute the reaction force by a compliance method. This method is applicable with respect to the dynamical model and furthermore is a rapid computation method, which is in the scope of our objective to realize "real-time" simulations.

\footnotetext{
${ }^{\dagger}$ OpenMASK, which stands for Modular Animation and Simulation Kit, is delivered as OpenSource Software: http://www.openmask.org
} 


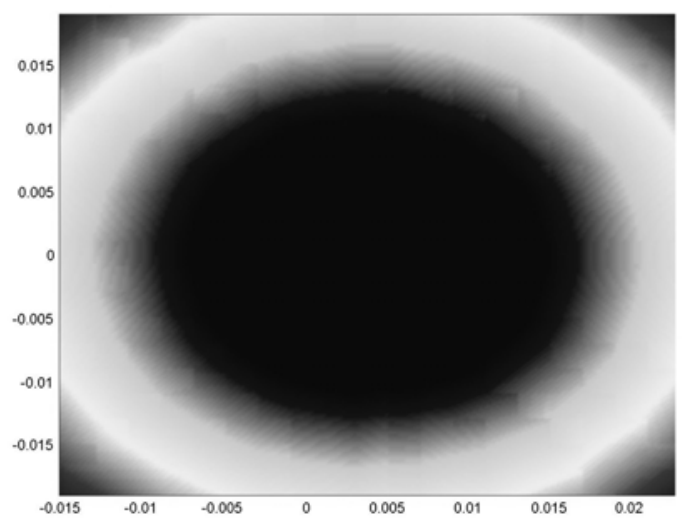

Figure 6: Pre-processed Medical database (one slice).

The proposed method for interaction treatment consists in three steps:

- As geometrical detection is obviously time consuming, the first stage consists in a preprocessing method to build, from the rough MRI (Magnetic Resonance Imaging) medical database, an interpolated distance cartography in the space which is presented, for a slice of the duct, in Fig. 6;

- The second stage of the reaction force computation is ensured by geometric collision detection. The links of the endoscope model are represented by interaction points for which collision is tested by calculating the distances from each interaction point to each "voxel" of the human duct model. This determines the distance from the point on the devices to the duct.

In case of collision, a classical compliance effort is applied to the link, which is proportional to the penetration depth and to the penetration velocity. Let dist be the penetration depth computed during the second stage, $\vec{n}$ be the inward normal to the duct model, $k$ be the global elasticity module of the duct and $f$ be the global viscosity of the duct.

The expression of the interaction effort is then: $\vec{F}=-k \bullet \operatorname{dist} \bullet \vec{n}-f \bullet(\vec{v} \bullet \vec{n}) \bullet \vec{n}$. This model, which parameters need to be identified by experimental measurement, seems to be a good one with respect to the stiffness difference between the rigid catheter and the soft human ducts. A simulation result is presented in Fig. 7.

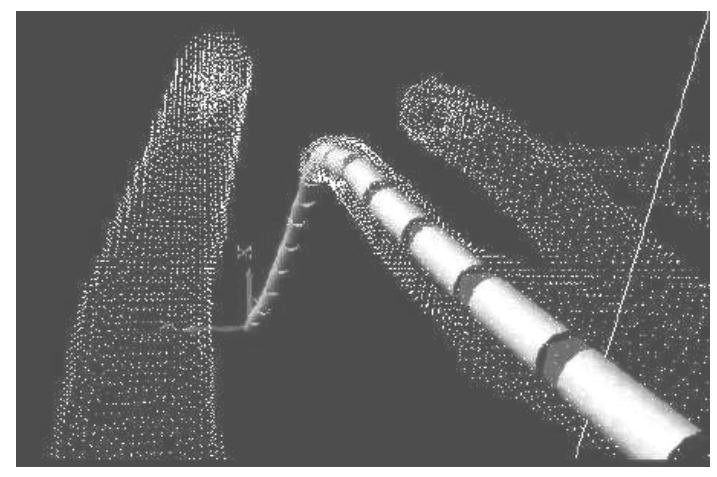

Figure 7: Insertion into a medical database.

\section{ENDOSCOPE COMMAND}

The ability to pilot the orientation of the links allows an easier introduction in the virtual duct and constitutes the major interest of using active devices. So, we have developed a module 
defining a controller, which commands the mechanical model. The controller is based on the geometrical description of the connections between two neighbouring links and on the behavioural characteristics of the SMA actuators, for which a model as been proposed in [15]. The behaviour of the controller for a 15 links catheter and for an orientation consign of $10^{\circ}$, illustrated in Fig. 8, shows a response time of $0.4 \mathrm{~s}$ and a good precision. The stability is good for the links located near to the head. The links located near to the basis shows oscillations, which are due to the important inertia supported.

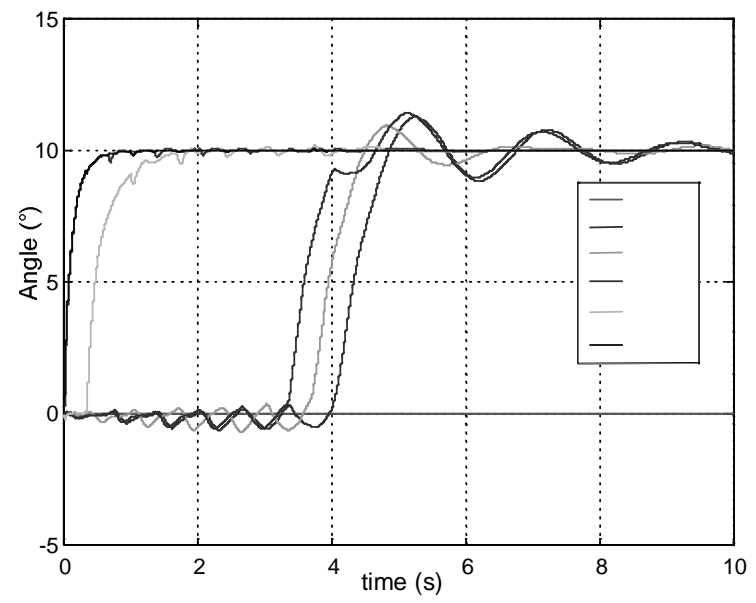

Figure 8: Response time.

To take benefit of the active property of the catheter, a command strategy is proposed. Its objective is to automatically adapt the endoscope curvature to the shape of the inspected ducts. In the real world, this will allow to minimise the contact between the endoscopic device and the inspected channel, in order to minimise wound during the operation. In Fig. 9, we present a simulation with a pushed device without any joint control: the applied forces onto the duct, represented by the red vectors, are very important.

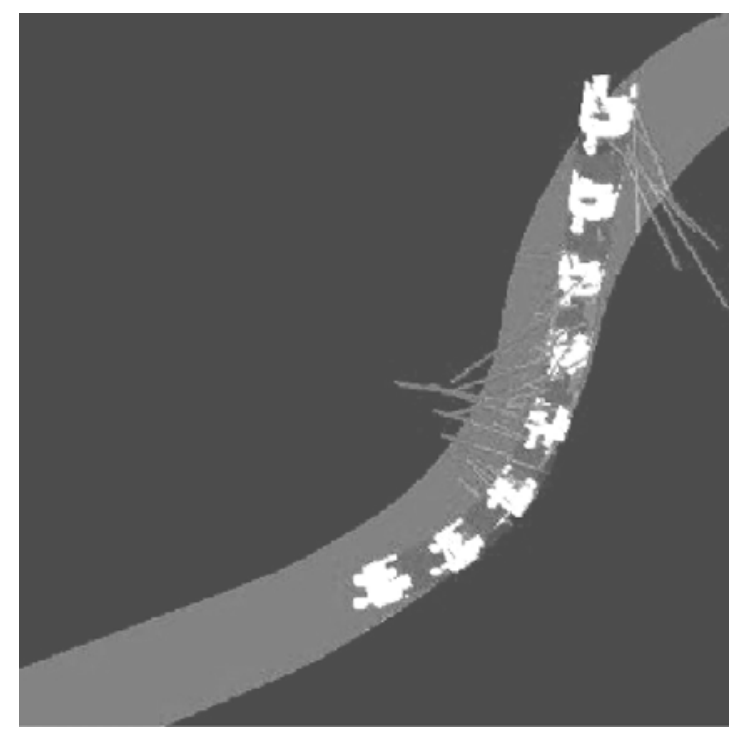

Figure 9: Simulation without joint control.

The used command strategy is based on a multi agent approach. It consists in splitting the steering mechanism into independent sub-systems, which constitutes the agents [16]. This distributed modular solution is independent from the structure length and should minimise the 
amount of information exchanged between the agents. The principle, presented in Fig. 10 for a two dimensional model, is quite simple. If an effort is detected on one of the links, which, in real world, will be achieved by piezoelectric film sensors, an orientation consign is applied to the preceding joint so as to decrease this interaction effort, and the opposite consign is affected to the next joint. The effect is, on one hand, to minimise the contact effort and, on the other hand, to ensure an unchanged orientation of the endoscope head. Numerical tests on stability show that the agent should be constituted of at least two consecutive links in the bidimensional case. The three-dimensional extension consists, because of the relative orientations of the pin joints, in agents constituted of four links.

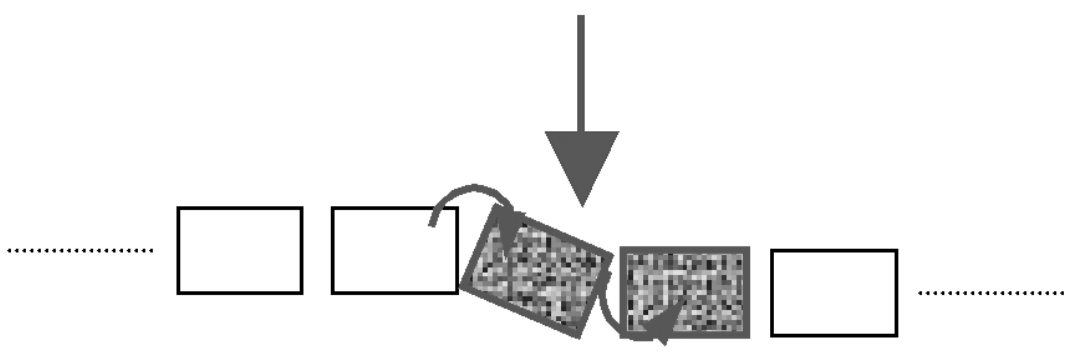

Figure 10: Multi-agent approach (2D principle).

An endoscope piloted with this strategy is proposed, for a three-dimensional model, in Fig. 11. This method, based on the contact detection, seems to be adapted to human ducts inspections and strongly limits the interaction efforts.

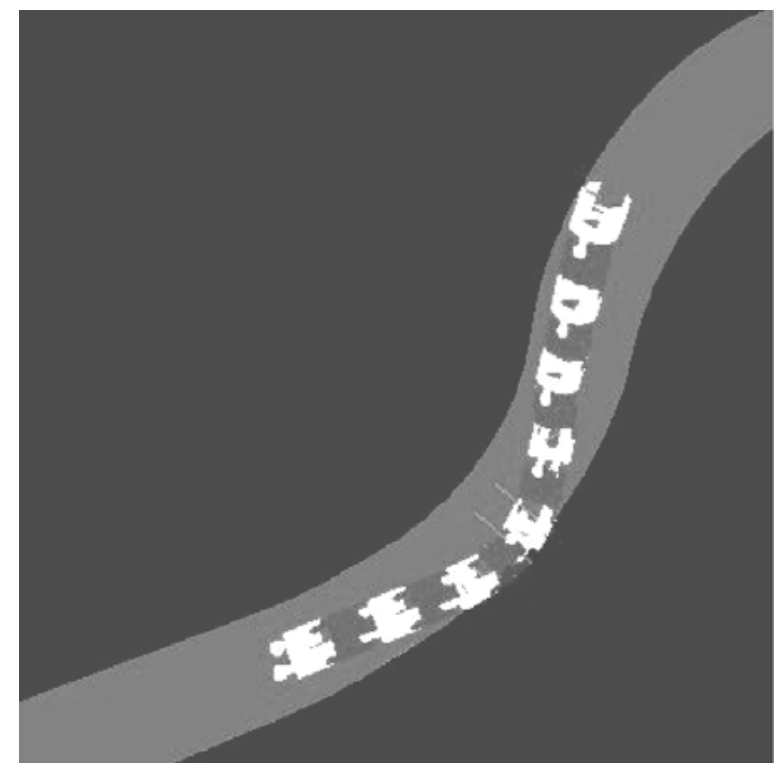

Figure 11: Result with a multi-agent control.

\section{OPTIMIZATION BY GENETIC ALGORITHMS}

The techniques of virtual prototyping [17] aim at improving the quality of the developed prototypes. In order to test the product functionalities, which could not easily be tested on real prototypes, we use the above described simulation process. The quality of the model, with respect to the task to accomplish (for example: a navigation task) can be measured by an objective function [18]. This function could take into account the developed energy to accomplish the task, which represents the comfort of the surgeon during the operation and the 
magnitude of the developed efforts during contact phases, which represents the security of this operation. In order to minimise the objective function, which leads to a better device, we use genetic algorithms [19] that have a good solution space exploration capability and lead to acceptable design solutions. Genetic algorithms imitate the natural process of species evolution [20]: an individual survives only if it can adapt to the surrounding environment. When it reproduces, its genes are transmitted to its descent. Among these genes, some mutates naturally.

The whole involved process is presented in Fig. 12 and the algorithm is defined as follow:

- Initialisation of the population, this step is performed only once;

- The evaluation of each candidate is done by performing a simulation in order to evaluate the objective, or cost, function;

- The best candidates are selected, by using partially unpredictable selection. This allow to keep mainly the candidates with a good performance, with respect to the specified task, but furthermore to have less good candidates which could lead to good descent;

- Among the so obtained population, the crossover is performed in order to generate a new population being intended in replacing the first one;

- In order not to impoverish the genetic content of the population a non determinist mutation is performed;

- The obtained population constitutes the initialisation of the next algorithm step;

- Convergence criterion may be defined as the number of generation, which ensures that the algorithm stops but does not ensure a strict convergence. Nevertheless, this test shows good results. Another criterion may be based on the average evolution of the population quality between to steps, insuring controlled convergence with inconvenience of possibly being endless.

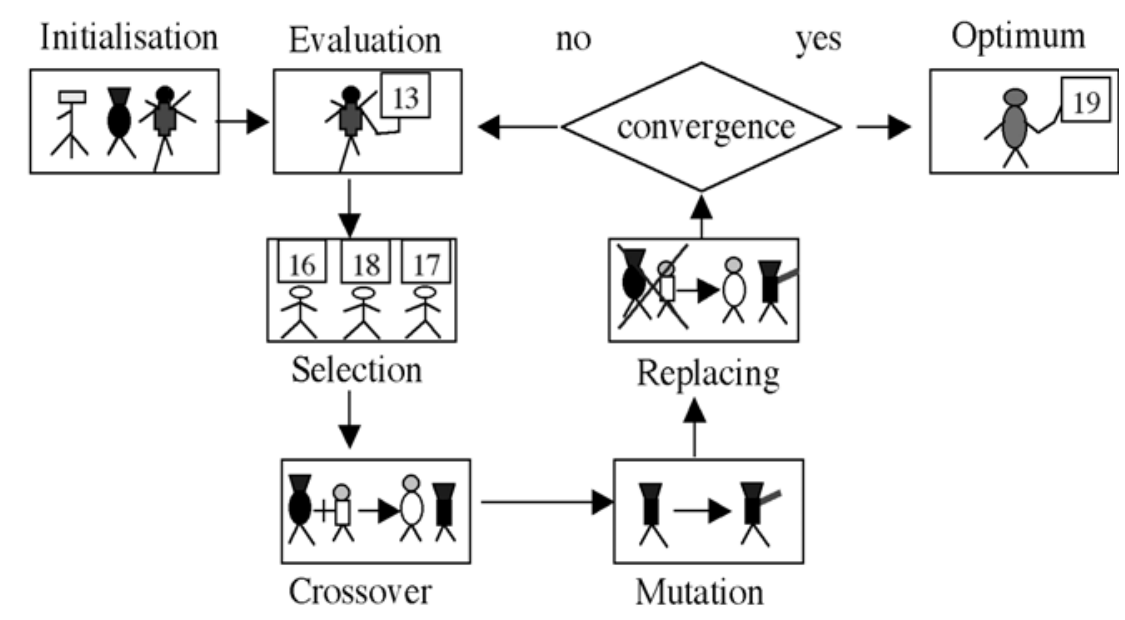

Figure 12: Genetic algorithms.

As usual with optimization processes, we have to define optimization variables: for the catheter case, with the modular proposed structure, we can choose the length of each link, the number of used links. This could lead to conceive the best prototype for one given inspection task. As the computation time, involved by this method, is obviously a punishing factor, leading to limit the investigation domain, we have chosen to define various complexity optimization levels:

- The first level consists in a purely geometrical analysis: at this step, for which a result into a 3D-duct model is presented in Fig. 13, we use an objective function built on the length and the number of links. 


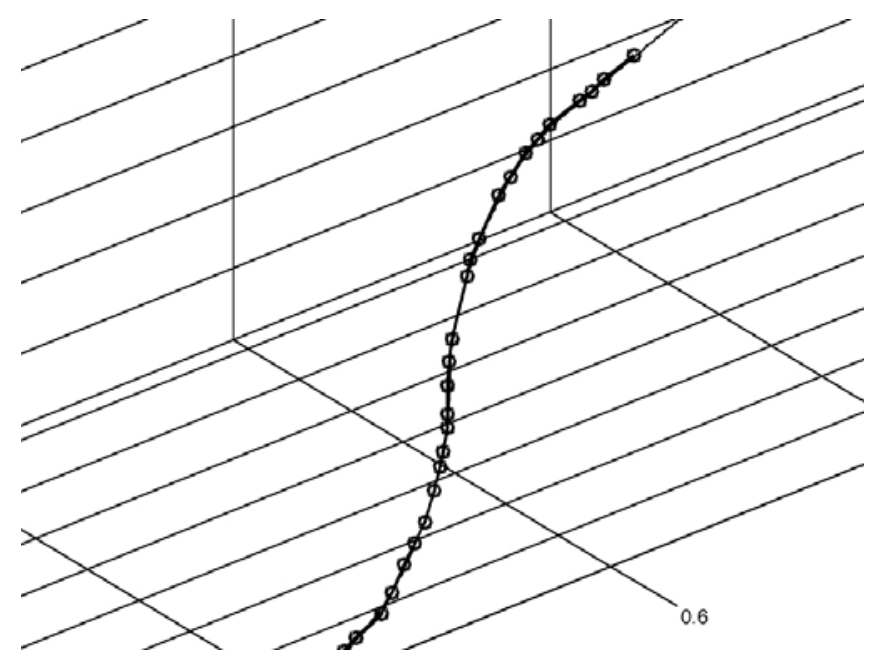

Figure 13: Result for 3D geometrical optimisation.

During the progression, the endoscope should interact at least with the patient tissues, so the average line of the channel to inspect defines the trajectory to follow. For this given trajectory, the algorithm aims at defining a prototype, which presents a good following capability, with a minimum number of links. Thus the objective function is defined by:

$$
f=\operatorname{good} \bullet i \bullet \exp ^{-k \bullet d i s t}
$$

In this equation, $i$ represents the index of the highest trajectory point reached by the endoscope head. It is saturated with the point index corresponding to the desired position. dist corresponds to the sum of the gaps from endoscope to trajectory during progress. good is a function, represented in Fig. 14, increasing the endoscope quality when it has a small number of links in order to decrease the complexity of the structure: $\operatorname{good}=\frac{2}{\pi}\left(\pi-\arctan \left(k \bullet\left(n_{\text {artic }}-n_{\max }\right)\right)\right)$.

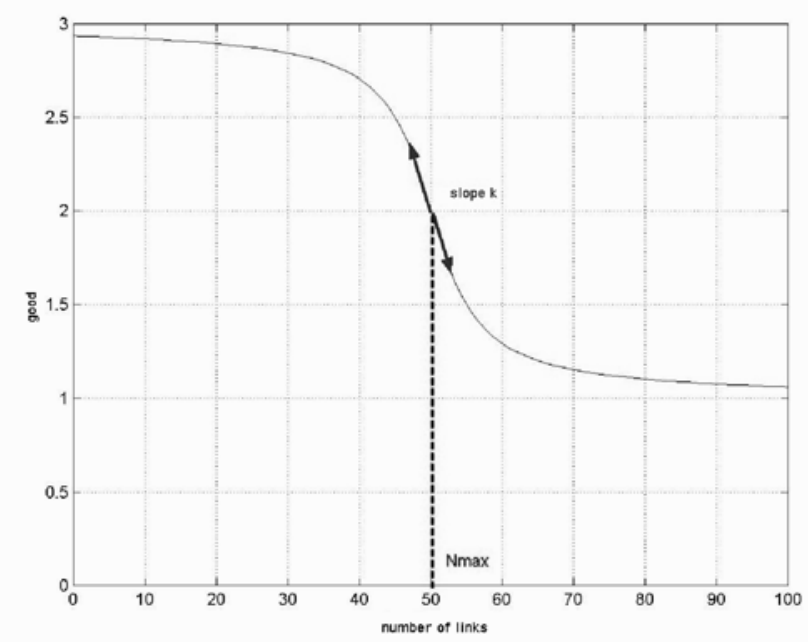

Figure 14: Improvement function.

- The second level is a degraded mechanical optimisation: at this stage, the good candidates issued from the first step, present a good conformation to the trajectory. The purpose is here to determine the actuators parameters insuring a good follow-up of trajectory. It is called a degraded mechanical simulation in the sense that the mechanical model of the 
device is used but no interaction with the duct is taken into account. This is the purpose of the third level;

- The third level should lie on a complete mechanical optimization: at this step, we dispose of models, which have the ability to follow correctly the predefined trajectory, avoiding the contact. The complete simulation is initialized with the lengths obtained during the first stage and with the mechanical and actuators parameters resulting from the second one. The interaction model is then introduced, and the objective function is the one related to the task to accomplish. It should lead to the best endoscope for this task and furthermore could take into account the mobility of the inspected duct.

In the presented optimization result (Fig. 13), each generation is composed of 40 individuals, and the computation is carried out on 80 generations. This number is chosen as convergence criterion for the optimization process. The optimization variable is the length of each link, which can be chosen between 4 to $18 \mathrm{~mm}$, by step of $2 \mathrm{~mm}$ (coded on $3 \mathrm{bits}$ ). The number of used links is variable. The computation time, using an $800 \mathrm{MHz}$ Pentium III with $256 \mathrm{Mb}$ of RAM is around 3 minutes. The optimal solution presented is a 31 segments endoscope and the maximum distance from links to the trajectory is $5 \mathrm{~mm}$. This optimization stage gives satisfactory results: indeed, the procedure does not find the trivial solution which is the endoscope made up of the segments of the smallest size, which solution is the closest to the trajectory but which results in an endoscope having a great number of segments.

\section{CONCLUSION AND FUTURE WORKS}

The simulation with multi-agent controller, presented in Fig. 11, proves the validity of this approach for minimizing contacts into the ducts, furthermore it can be extended to the case where the ducts are not rigid but elastically, or visco-elastically deformable, as in reality [11, 21]. A sigmoid untwisting, that is a classical examination in coloscopy, has been reproduced for testing the simulator. The main part of this examination is done when the endoscopic device is withdrawed. The medical practitioner try to reach the ileocolic valve: this part is very delicate because the practitioner has to align the endoscope with the colon in order to continue the progression. Furthermore, the colic angles are very difficult to cross. The final point is the crossing of the sigmoid colon. The technique consists in rolling up the endoscopic device into the sigmoid. Then the practitioner unrolls the buckle by rotating the endoscopic device and by withdrawing it. The sigmoid is untwisted and the rest of the inspection is more easily done. An extract of the simulation is presented in Fig. 15, shows that this operation can be reproduced thanks to the developed simulator.

We have developed a simulator allowing to compute the progression of a poly-articulated endoscope, based on an under development real prototype, actuated by SMA actuators with identified behaviour model. This simulator is based on a mechanical description of the device and on the interacting environment, specific to the considered patient, through a medical MRI acquisition. As mentioned in the introduction, an experimental work is to be done in order to identify the contact model parameters.

Different control strategies for the actuators have been proposed, which results have been presented and which give good results to minimize the contact between the catheter model and the patient database.

In order to improve the accuracy of the considered prototypes, the use of the simulator seems to be a good direction especially when coupled to optimization process. We have proposed different optimization approaches by genetic algorithms and developed some results. Geometrical optimization gives cheering results: it has been shown to predetermine endoscope segments lengths. 


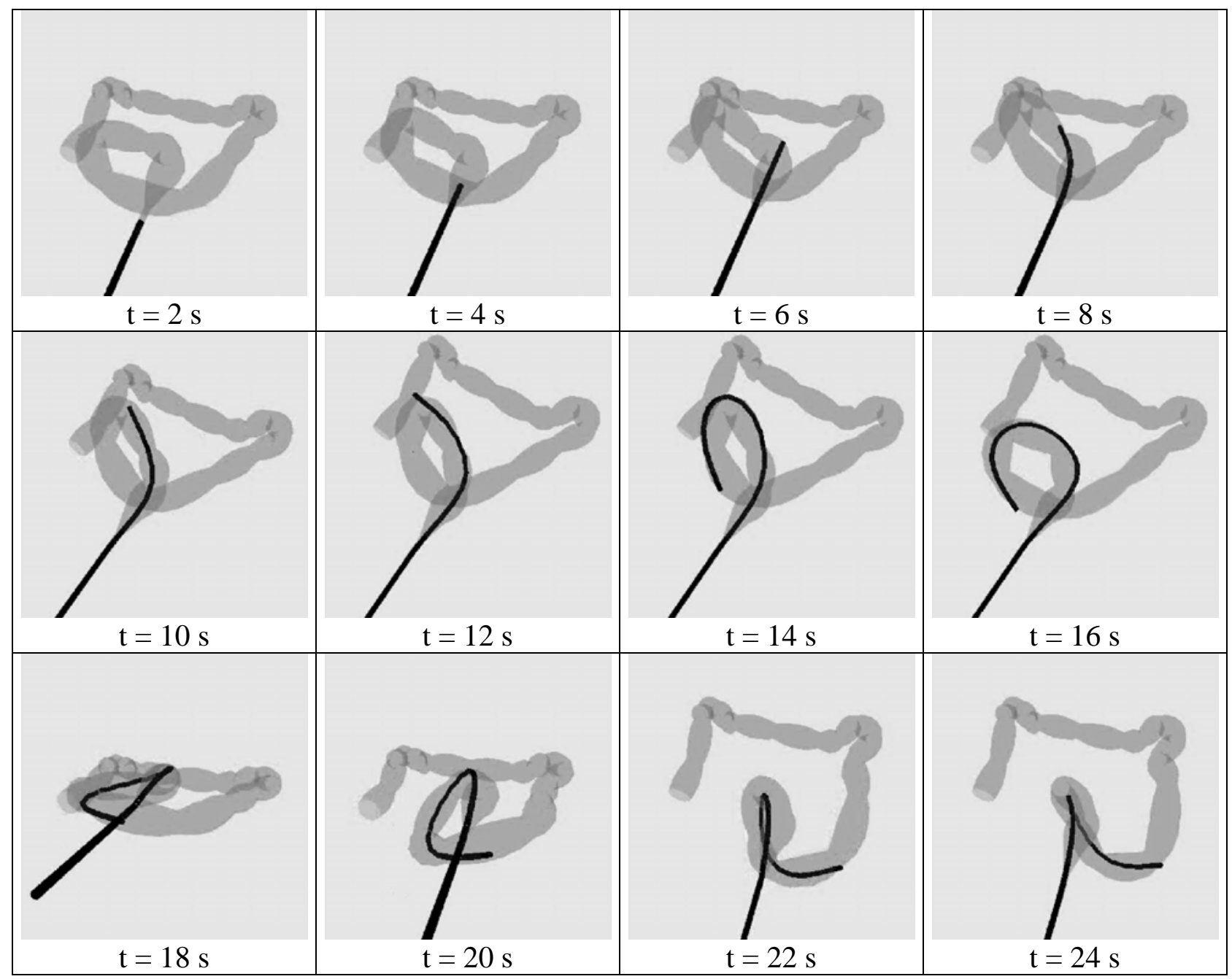

Figure15: Sigmoid untwisting.

The second stage of degraded mechanical optimization should allow identifying mechanical parameters adapted for the actuators choice. This optimization procedure will be very similar to the geometrical optimization one, last generation of which will be used to initialize this second stage. Springs and damps will be added to the already calculated lengths. They will be evaluated with criteria taking into account the displacements, the eigen frequencies and the damping in order to obtain satisfactory within the minimum of generation steps, since here time of test for each individual will be considerably increased. Then, they will be generalized to the whole system parameters, as segments diameter or thrusts value, in order to be able to propose the most powerful structure to the surgeon.

The lake of interfacing capabilities, especially in the haptic domain, needs to be examined. It is expected that such devices should improve the control over the catheter in order to define "real" tasks to accomplish in real time. This would also allow feeling the contact between the catheter and the ducts in order to produce a training simulator.

\section{ACKNOWLEDGEMENT}

This work was partially supported by the Centre National de la Recherche Scientifique, France (CNRS/SPI). 


\section{REFERENCES}

[1] Cohn, M.; Crawford, L.; Wendlandt, J.; Sastry, S. S. (1995). Surgical applications of milli-robots. Journal of robotic systems, Vol. 12, No. 6, 401-416

[2] Lim, G.; Minami, K.; Yamamoto, K.; Sugihara, M.; Uchiyama, M.; Esashi, M. (1996). Multi-link active catheter with snake-like motion, Robotica, Vol. 14

[3] Dumont, G.; Chapelle, F.; Bidaud, P. (2001). Toward virtual prototyping of active endoscopes, Proceedings of ISR2001 (International Symposium on Robotics), IFR (International Federation of Robotics), Seoul, Korea, 821-826

[4] Ikuta, K.; Iritani, K.; Fukuyama, J. (2001). Mobile virtual endoscope system with haptic and visual information for non-invasive inspection training, Proceedings of ICRA 2001 (International conference on robotics and automation), IEEE, Seoul, Korea, 2037-2044

[5] Takehana, S.; Ueda, Y.; Gotanda, M.; Sakurai, T.; Adachi, H. (1990). Apparatus for bending an insertion section of an endoscope using a SMA, United States Patent 05/06/1990 US4930494

[6] Montesi, M. C.; Martini, B.; Pellegrinetti, A.; Dario, P.; Lencioni, L.; Montano, A. (1995). An SMA-based flexible active endoscope for minimal invasive surgery, Journal of micromechanic and microengineering, Vol. 5, 180-182

[7] Park, K.; Esashi, M. (1999). An active catheter with integrated circuit for communication and control, Technical Digest of the Twelfth IEEE International Conference On Micro Electro Mechanical Systems (MEMS '99), IEEE, 400-405

[8] Szewczyk, J.; Sars, V. D.; Bidaud, P.; Dumont, G. (2000). An active tubular polyarticulated micro-system for flexible endoscope, Proceedings of ISER2000 (7th International Symposium on Experimental Robotics), Hawaii

[9] Kühl, C.; Dumont, G.; Mognol, P.; Gouleau, S.; Furet, B. (2002). Active catheter prototyping: From virtual to real, Proceedings of 4th International Conference On Integrated Design And Manufacturing In Mechanical Engineering, Primeca, IFMA, Clermont-Ferrand, France

[10] Dumont, G.; Kühl, C.; Bidaud, P. (2002). Simulating and optimizing active endoscope prototypes, Proceedings of ISR2002 (International Symposium on Robotics), IFR (International Federation of Robotics), Stockholm, Sweden

[11] Kühl, C. (2003). Prototypage virtuel d'endoscopes à actionneurs distribués, PhD thesis, École normale supérieure de Cachan

[12] Press, W. H.; Teukolsky, S. A.; Vetterling, W. T.; Flannery, B. P. (1992). Numerical recipes in $C$, The art of scientific computing, $2^{\text {nd }}$ edition, Cambridge University Press

[13] Margery, D.; Arnaldi, B.; Chauffaut, A.; Donikian, S.; Duval, T. (2002). Openmask: Multithreaded or modular animation and simulation kernel or kit: a general introduction, Proceedings of VRIC, 101-110, http://www.openmask.org

[14] Fung, Y. C. (1984). Biomechanics, Mechanical Properties of Living Tissues, Springer Verlag

[15] Troisfontaine, N.; Bidaud, P. (1998). Position and force control of sma micro-actuators, International Advanced Robotics Programm (IARP), 110-126

[16] Duhaut, D. (1993). Using a multi-agent approach to solve the inverse kinematics, Proceedings of IROS 1993 (Intelligent Robot and System Conference), 2002-2007

[17] Dumont, G.; Kühl, C. (2005). Finite element simulation for design optimization of shape memory alloy spring actuators, Engineering Computations, International Journal For Computer Aided Engineering And Software (accepted for publication)

[18] Endo, K.; Maeno, T. (2001). Simultaneous generation of morphology of body and neural system of multi-linked locomotive robot using evolutionary computation, Proceedings of ISR2001 (International Symposium on Robotics), IFR (International Federation of Robotics), Seoul, Korea, 499-504

[19] Wall, M. (1996). Galib: a C++ library of genetic algorithm components, Technical report, Massachusetts Institute of Technology, Mechanical Engineering Department

[20] Darwin, C. (1859). The origin of species

[21] Kühl, C.; Dumont, G. (2005). Coloscopy simulation : toward endoscope improvement. Journal of Computer Methods In Biomechanics And Biomedical Engineering, Special issue 'Computeraided medicine and surgery' (to appear 2005) 